\title{
Avaliação do efeito do tratamento de distúrbios temporomandibulares sobre o zumbido
}

\section{Evaluating the effect of the temporomandibular disorder treatment over tinnitus}

\author{
Guilherme Webster', Cláudio Márcio Yudi lkino², Bertholdo Werner Salles³, Aline da Rocha Lino ${ }^{4}$ Evandro Maccarini Manoel${ }^{5}$, \\ Waldir Carreirão Filho'.
}

1) Residente em Otorrinolaringologia no Hospital do Servidor Público Municipal de São Paulo. Médico Residente.

2) Doutor em Otorrinolaringologia pela Universidade de São Paulo. Professor Adjunto do Departamento de Cirurgia da UFSC

3) Doutor em Odontologia pela Universidade Federal de Santa Catarina. Professor Adjunto do Departamento de Odontologia da UFSC

4) Residente em Clinica Médica no Hospital Regional de São José Dr. Homero de Miranda Gomes. Médica Residente.

5) Residente em Otorrinolaringologia na EPM-UNIFESP. Médico Residente.

6) Mestre em Otorrinolaringologia pela Pontifícia Universidade Católica do Rio de Janeiro. Professor Adjunto do Departamento de Cirurgia da UFSC

Instituição: Universidade Federal de Santa Catarina - UFSC

Florianópolis / SC - Brasil.

Endereço para correspondência: Guilherme Webster - Rua Castro Alves, 355 - Bairro aclimação - São Paulo / SP - Brasil - CEP: 01532-001 - Telefone: (+55 11) 6953-9011-

E-mail: guilhermewebster@uol.com.br

Artigo recebido em $1^{\circ}$ de Abril de 2011. Artigo aprovado em 21 de Maio de 2011

\section{RESUMO}

Introdução: A interação entre o zumbido e os distúrbios temporomandibulares é um tema que possui abordagem antiga e complexa, pois os fatores etiológicos, bem como a patogênese desta inter-relação ainda não são bem definidos. Ademais, sabe-se que o zumbido possui maior prevalência nos portadores de distúrbios temporomandibulares quando comparados com a população geral, o que sugere haver esta correlação.

Objetivo: Avaliar o efeito do tratamento dos distúrbios temporomandibulares na percepção do zumbido.

Método: Trata-se de um estudo de coorte, prospectivo, em que se estudaram pacientes portadores de distúrbios temporomandibulares (DTM) que apresentavam zumbido antes e após o tratamento odontológico do DTM. Os pacientes foram avaliados quanto à idade, sexo, caracterização do zumbido - local do sintoma e tempo de duração e avaliação auditiva através de audiometria. A intensidade do zumbido foi avaliada através de escala analógico-digital antes e após o tratamento dos DTM.

Resultados: Avaliamos 15 pacientes com DTM e zumbido, com idade média de $37,7 \pm 17,1$ anos, sendo $86,7 \%$ do sexo feminino. Em 60\% dos casos o zumbido era unilateral e a mediana do tempo de duração foi de 24 meses. Em 5 (33,3\%) pacientes identificou-se perda auditiva neurossensorial a audiometria. Comparando-se os escores da escala analógico-visual antes e após o tratamento odontológico, verificou-se que houve redução significativa $(\mathrm{p}<0,001)$ da intensidade do zumbido. Em 4 (26,6\%) pacientes houve desaparecimento do zumbido.

Conclusão: Houve redução significativa na percepção do zumbido nos pacientes submetidos a tratamento das desordens temporomandibulares.

Palavras-chave: transtornos da audição, zumbido, transtornos da articulação temporomandibular, transtornos craniomandibulares.

\section{SUMMARY}

Introduction: The interaction between tinnitus and temporomandibular disorders is a very complex issue that has been long approached, because neither the etiologic factors nor the pathogenesis of such a two-way association has been clearly defined yet. Additionally, tinnitus is known to be more prevalent in temporomandibular dysfunction individuals in comparison with the general population, hence, suggesting the existence of this two-way association.

Objective: Evaluate the effect of the temporomandibular disorder treatment when tinnitus is noticed.

Methods: It is about a prospective cohort study, in which temporomandibular dysfunction (TMD) individuals showing a tinnitus before and after the dental TMD treatment were studied. Patients' age, sex, and tinnitus features - place of symptom and time length were examined, and an audiometric evaluation was performed. Intensity of tinnitus was evaluated by a digital analogue scale before and after the TMD treatment.

Results: We evaluated 15 TMD and tinnitus patients aged between $37.7 \pm 17.1,86.7 \%$ of whom were female. In $60 \%$ of the cases, tinnitus was unilateral and the average time length was 24 months. In 5 (33.3\%) patients, a neurosensorial hearing loss was seen at audiometry. When comparing the visual analogue scale scores before and after the dental treatment, a significant decrease $(p<0,001)$ in the intensity of tinnitus was evident. In 4 (26.6\%) patients, tinnitus disappeared.

Conclusion: A significant decrease in the recognition of tinnitus by patients submitted to a temporomandibular dysfunction treatment was evident.

Keywords: hearing disorder, tinnitus, temporomandibular joint dysfunction, craniomandibular disorders. 


\section{INTRODUÇÃO}

Zumbido é a percepção do som sem fonte de estímulo acústico externa. Possui alta prevalência, atingindo aproximadamente 50 milhões de pessoas nos Estados Unidos (1). Na Europa, esse valor é estimado entre $7 \%$ a $14 \%$ da população, sendo que dentre essas pessoas, $1,0 \%$ a $2,4 \%$ possui zumbido grave e incapacitante (1). Sabe-se que o zumbido é um sintoma, estando presente em diversas afecções clínicas (1).

As disfunções Temporomandibulares (DTM) são um termo que envolve muitos problemas clínicos que acometem os músculos mastigatórios, a articulação temporomandibular e estruturas associadas ou ambos $(2,3)$. Os DTM são muito comuns na população, estudos mostram que mais de $50 \%$ da população apresenta com um ou mais sinais de DTM (4-6). No entanto, apenas 3,6\% a 7,0\% necessitam de algum tipo de tratamento (6).

A interação entre os sintomas otológicos - tais como o zumbido - e as DTM é um tema que possui abordagem antiga (6-9), complexa e associada a diferentes áreas, como a medicina e a odontologia. Tais fatos podem ser comprovados com trabalhos como os de Costen (1934) (9), bem como trabalhos atuais como os realizados por RAMIREZ et al $(2005,2007)(10-11)$. Em pacientes portadores de DTM, a presença de zumbido oscila entre 50 a $75 \%(5,12-$ 14), o que evidencia uma prevalência maior nesses indivíduos quando comparados com a população geral $(5-6,15)$.

Os fatores etiológicos, bem como a patogênese desta inter-relação entre o zumbido e os DTM ainda não são bem definidos $(6,8,12)$, tendo então aparecido diversas teorias que buscam justificar esta correlação, como a que menciona a existência de uma relação anatômica entre o aparelho auditivo e o aparelho estomatognático (10-12). Porém, como os DTM são frequentes, nada impede que estes mesmo pacientes apresentem outras afecções que também sejam causa de zumbido.

Assim, este estudo tem por objetivo avaliar, através de escala semi-quantitativa, o evento do tratamento dos DTM sobre o zumbido, independente de sua etiologia.

\section{MéTOdo}

O projeto deste trabalho foi enviado ao Comitê de Ética e Pesquisas com Seres Humanos (CEPSH) da UFSC e aprovado sob o parecer número 213/08.

Trata-se de um estudo do tipo longitudinal, prospectivo, não controlado, o qual foi realizado no ambu- latório de oclusão do curso de graduação em Odontologia da Universidade Federal de Santa Catarina e no serviço de Otorrinolaringologia e Cirurgia de Cabeça e Pescoço do Hospital Universitário da Universidade Federal de Santa Catarina.

Para a confecção do presente estudo foram selecionados os pacientes, com idade superior a 18 anos admitidos para início de avaliação no ambulatório de oclusão do curso de graduação em Odontologia da Universidade Federal de Santa Catarina, durante o período compreendido entre 08 de outubro de 2008 e 23 de agosto de 2009, voluntários a participação da pesquisa.

Foram incluídos no presente trabalho os pacientes que: 1) Concordaram com o termo de consentimento livre e esclarecido 2) Apresentavam maioridade civil; 3) Possuíam distúrbio temporomandibular caracterizado por especialista da área; 4) Possuíam o sintoma de zumbido e 5) Não tinham iniciado o tratamento odontológico para DTM até o momento da primeira entrevista. Foram excluídos os pacientes que: 1) Desistiram da participação da pesquisa; 2) Não realizaram o exame audiométrico; e 3) Não terminaram o tratamento proposto pelo profissional da área de odontologia.

Num primeiro momento, ocorrido na consulta de admissão, realizou-se uma anamnese dirigida através de um questionário, no qual foram avaliados a idade do paciente, o sexo, há quanto tempo o paciente apresenta zumbido, caracterização do zumbido - local do sintoma e grau de intensidade, a qual foi medida através da apresentação de uma escala analógico-visual ao paciente (Figura 1), exposição a ruídos (hábito de ir a danceterias, shows musicais ao vivo, usar fones de ouvido com som intenso, exposição no trabalho e uso de equipamentos de proteção individual-EPI), comorbidades (hipertensão arterial sistêmica - HAS -, diabetes mellitus - DM-, cirurgia otológica prévia, internações, contato com drogas ototóxicas). Em seguinte, realizou-se a otoscopia bilateral, rinoscopia anterior e oroscopia. Por fim, foi definido o diagnóstico odontológico da DTM, sendo que o diagnóstico e o tratamento proposto foram anotados e, ao finalizar o questionário, foi solicitada

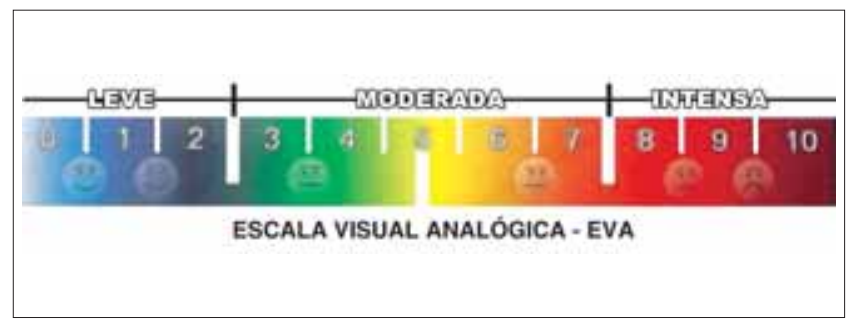

Figura 1. Escala analógico-visual. 
uma audiometria tonal e vocal que se realizou em outra oportunidade, desde que prévia ao início do tratamento.

Após o término do tratamento odontológico para DTM - o qual tinha uma duração média de 5 meses -, realizou-se a segunda avaliação proposta, que consistiu na mensuração da intensidade do zumbido através da mesma escala analógico-visual da avaliação inicial. No caso da presença de rolha de cerume, esta foi removida antes da aplicação da escala.

Para a realização da audiometria, foi utilizado o aparelho modelo SIBELMED AC-50D em cabina acústica, com o uso de fone de ouvido e vibrador ósseo, avaliando-se os limiares auditivos por via aérea nas frequências de 250, 500, 1000, 2000, 3000, 4000, 6000 e 8000 Hz e por via óssea nas frequências de 500, 1000, 2000, 3000 e 4000 Hz.

Para a analise dos dados, a mesma foi expressa na forma de números absolutos e porcentagens. O cálculo das médias e desvio-padrão, bem como a confecção dos gráficos e tabelas apresentados foi realizada através da ferramenta Microsoft Excel 2007. Para análise dos valores da escala analógico-visual antes e após o tratamento, utilizou-se o Teste de Wilcoxon Signed Rank, do software GraphPad Prism 4 for Windows, versão 4.02. Adotou-se o nível de significância ( $p)$ de 95\% ( $p<0,05)$, conforme os padrões utilizados em estudos biológicos.

\section{RESULTADOS}

No presente estudo, a amostra inicial era de 42 pacientes, contudo - ao aplicar os critérios de exclusão, principalmente o de desistência do tratamento odontológico e a não realização da audiometria -, restaram apenas 15 pacientes.

Com isso, avaliaram-se 15 pacientes que apresentavam distúrbios temporomandibulares e zumbido, durante o período compreendido entre 08 de outubro de 2008 e 23 de agosto de 2009. A idade média foi de 37,73 $\pm 17,11$ anos, variando de 18 a 73 anos. Na Figura 2 apresentamos a distribuição por sexo.

O tempo de zumbido apresentou mediana de 24 meses, percentil 25 de 19 meses e percentil 75 de 72 meses. O zumbido era unilateral em $9(60,0 \%)$ pacientes e bilateral em $6(40,0 \%)$ pacientes. Dos pacientes com zumbido unilateral, o lado mais acometido foi o direito $(66,6 \%)$.

Na Tabela 1 apresentamos os pacientes segundo a idade, sexo e intensidade do zumbido, antes e após tratamento da DTM, aferida pela escala analógico-visual.

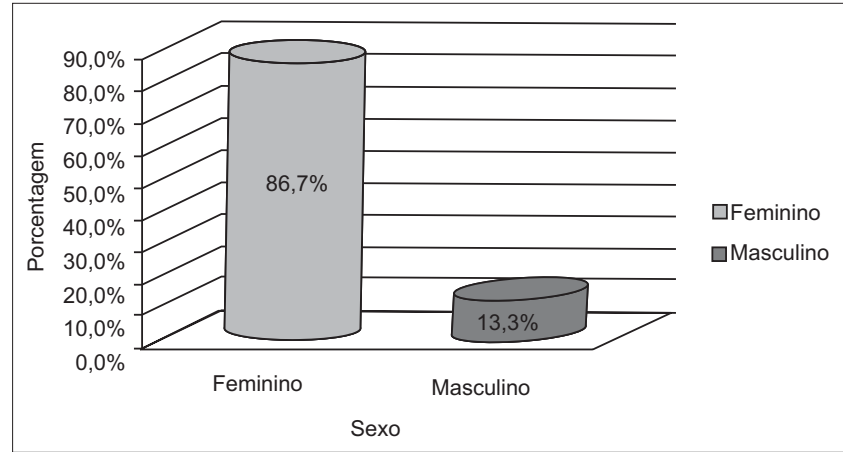

Figura 2. Distribuição dos pacientes segundo o sexo.

Tabela I. Descrição da população estudada.

\begin{tabular}{lcccc}
\hline Identificação & Idade & Sexo $^{\text {a }}$ & $\begin{array}{c}\text { Escore pré } \\
\text { tratamento }\end{array}$ & $\begin{array}{c}\text { Escore Pós } \\
\text { tratamento }\end{array}$ \\
\hline J.S.S & 25 & Fem & 5 & 1 \\
D.R.R.S & 40 & Fem & 7 & 4 \\
E.M.H & 23 & Fem & 2 & 0 \\
A.C & 26 & Fem & 2 & 0 \\
R.A.S & 52 & Masc & 8 & 2 \\
A.D & 37 & Fem & 4 & 1 \\
C.M & 18 & Fem & 8 & 2 \\
L.M.C & 27 & Fem & 5 & 0 \\
C.M & 22 & Fem & 2 & 0 \\
E.B.S.L. & 55 & Fem & 7 & 1 \\
J.M & 50 & Fem & 9 & 1 \\
P.S.B & 29 & Fem & 10 & 3 \\
J.C.V & 73 & Masc & 9 & 6 \\
K.W.M & 25 & Fem & 5 & 4 \\
M.K & 64 & Fem & 10 & 8 \\
\hline
\end{tabular}

a Fem $=$ Feminino; Masc $=$ Masculino

Comparando os escores antes e após o tratamento odontológico através do teste de Wilcoxon Signed Rank para amostras pareadas, verificou-se que houve redução significativa $(\mathrm{p}<0,001)$ da intensidade do zumbido. Em 4 (26,66\%) pacientes houve desaparecimento do zumbido.

Quanto à exposição de ruídos, 10 (66,66\%) pacientes não apresentaram exposição alguma, enquanto 5 (33,33\%) pacientes apresentaram-na. Dos expostos, 1 paciente relatou exposição crônica à danceteria, shows ao vivo e ouvir som intenso com fone de ouvido; 1 paciente apresentou exposição à danceteria e ouvir som intenso com fone de ouvido; 1 paciente apresentou apenas exposição à danceteria e, por fim, 2 pacientes relaram apenas exposição a ruídos no trabalho (confecção de portas e o outro em perfuração de asfalto).

Com relação as comorbidades, 3 (20,00\%) dos pacientes apresentaram HAS - sendo que 1 paciente estava em uso de atenolol apenas; 1 paciente utilizava 
Tabela 2. Descrição das alterações no exame físico.

\begin{tabular}{|c|c|c|c|c|}
\hline Identificação & Idade & $\mathrm{SexO}^{\mathrm{a}}$ & Rinoscopia & Otoscopia \\
\hline A.D & 37 & Fem & Desvio septalà direita & Semalterações \\
\hline J.C.V & 73 & Masc & Semalterações & Membranatimpânica retraídaà direita \\
\hline K.W.M & 25 & Fem & Hipertrofia de Cornetos inferiores & Semalterações \\
\hline M.K & 64 & Fem & $\begin{array}{c}\text { Desvio septalà esquerda }+ \text { Hipertrofia } \\
\text { de Cornetos inferiores }\end{array}$ & Semalterações \\
\hline
\end{tabular}

${ }^{a} \mathrm{Fem}=$ Feminino; Masc $=$ Masculino.

Tabela 3. Descrição das alterações na audiometria.

\begin{tabular}{lcccc}
\hline Identificação & Idade & Sexo $^{\text {a }}$ & $\begin{array}{c}\text { Valoresaudiométricos do déficit } \\
\text { auditivo neurossensorialà direita }\end{array}$ & $\begin{array}{c}\text { Valoresaudiométricos do déficit } \\
\text { auditivo neurossensorialà esquerda }^{\text {b }}\end{array}$ \\
\hline D.R.R.S & 40 & Fem & 8 & 3 \\
R.A.S & 52 & Masc & 8 & $\ldots$ \\
J.C.V & 73 & Masc & 3,4 e 8 & 3,4 e 8 \\
K.W.M & 25 & Fem & $1,2,3,4$ e 8 & I \\
M.K & 64 & Fem & 3 e 4 & $\ldots$
\end{tabular}

${ }^{\mathrm{a}} \mathrm{Fem}=$ Feminino; Masc $=$ Masculino.

b Aferido em número múltiplos de $10^{3}$ Hertz.

alfametildopa, hidroclorotiazida, enalapril e ácido acetilsalicílico; 1 paciente estava em uso de losartan. Não encontramos antecedentes de ototoxicidade, DM, cirurgia otológica prévia em nossa amostra.

No exame físico, $11(73,33 \%)$ pacientes não tinham quaisquer alterações, ao passo que em $4(26,66)$ pacientes foram verificadas as alterações descritas na Tabela 2.

Com relação a audiometria tonal, esta foi normal em $10(66,6 \%)$ pacientes, ao passo que em 5 (33,3\%) pacientes observou-se algum grau de perda auditiva, conforme descrito na Tabela 3 .

Na Figura 3 apresentamos a distribuição dos pacientes segundo os diagnósticos odontológicos dos DTM.

O tratamento dos distúrbios temporomandibulares em todos os pacientes foi à confecção de uma órtese dentária - placa de Michigan-de uso contínuo. Para os casos de desordens musculares com dor intensa associou-se antiinflamatório não hormonal (AINH) e fisioterapia, enquanto que no caso de iatrogênia, foi-se retirada a órtese dentária que estava causando o problema e, após, associou-se a placa de Michigan.

\section{Discussão}

A relação entre distúrbios temporomandibulares e os sintomas otológicos - como o zumbido - não é completamente estabelecida e elucidada $(1,3,7)$, contudo há indícios de que possa existir (1-2,5-7,11-13). Desde a

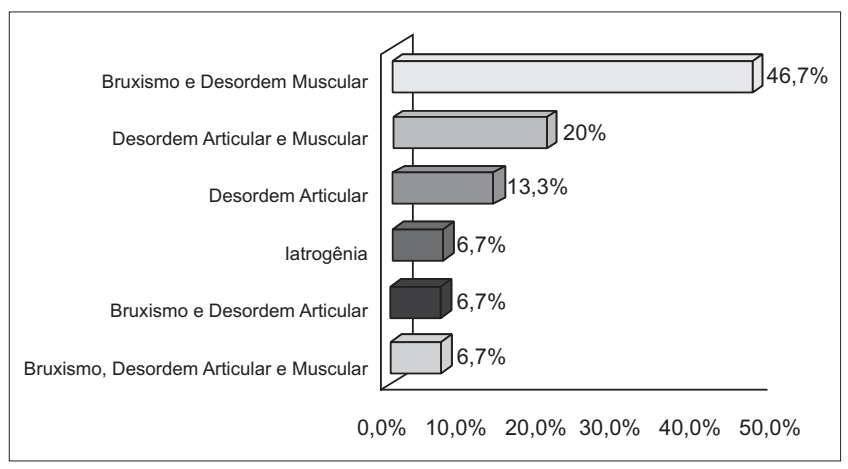

Figura 3. Distribuição dos pacientes quanto ao diagnóstico odontológico

primeira metade do século passado, procura-se estabelecer a etiologia dos sintomas aurais com a DTM, tendo-se verificado relações anatômicas, neurológicas, emocionais (5-6), sendo que a hipótese de multicausalidade ainda é a mais aceita nos meios científicos (12-14).

Conforme a literatura pesquisada, verificou-se que, na grande maioria dos estudos realizados em pacientes com zumbido e distúrbios temporomandibulares, houve um predomínio de acometimento no sexo feminino (1$2,11,13)$, da mesma forma que observamos.

Quanto às características do zumbido, no estudo de Parker e Chole (1995) (9) não foi verificado diferença significativa entre acometimento unilateral ou bilateral, ao passo que no nosso estudo, visualizamos um predomínio de ocorrência unilateral de zumbido. Já no que se refere ao tempo de acometimento, Wright e Bifano (1997) (15) 
apresentaram um tempo de duração que variava entre 4 meses até 40 anos, sendo que a nossa estatística encontrase em assentimento com o trabalho citado.

Com relação ao efeito do tratamento de distúrbios temporomandibulares sobre o zumbido, WRIGHT e BIfANO (1997) (15) avaliaram os pacientes portadores de DTM e zumbido, verificando uma melhora significativa na sintomatologia do zumbido após a realização de um tratamento do distúrbio temporomandibular que os acometia. Já em 1998, GaRANHANi (12) realizou uma revisão da literatura, na qual constatou que o tratamento conservador utilizado para o tratamento de distúrbios temporomandibulares traz resultados favoráveis na melhora dos sintomas otológicos, como o zumbido.

Wright e Bifano (2000) (14) em estudo com pacientes que apresentavam zumbido, vertigem e otalgia de causas não-otológicas e DTM observaram melhora do zumbido em $64 \%$ dos pacientes após o tratamento conservador dos DTM. Nós também observamos melhora em uma população semelhante, porém não excluímos pacientes com alterações audiométricas.

Há ainda o relato de caso de Tori e CHiwata (2007) (16), em que a paciente relatava sintomas aurais à direita (zumbido, otalgia, vertigem) de etiologia desconhecida, bem como dores severas à palpação na ATM direita, tendo seus sintomas resolvidos após tratamento conservador para DTM que a acometia.

De Felicio et al (2008) (10) procuraram avaliar a frequência e a relação entre os sintomas otológicos e os sinais e sintomas orofaciais dos DTM, bem como o efeito da terapia conservadora sobre os músculos orofaciais. Os autores obtiveram uma melhora do zumbido após o tratamento, sendo que o antes e depois eram, respectivamente, $60 \%$ e 20\% dos pacientes acometidos por zumbido, contudo os autores não mencionaram a forma com a qual graduaram os sintomas otológicos.

Já Bösel et al(2008) (17), em estudo com pacientes portadores de zumbido crônico e DTM, não observaram efeito do tratamento de DTM sobre o zumbido e, por conseguinte, concluíram que a existência de zumbido em pacientes com distúrbios temporomandibulares seria apenas coincidência.

Entretanto, URBAN et al (2009) (3), após revisão da literatura, observaram a existência de uma ligação significativa entre os sintomas otológicos e DTM, bem como uma diminuição desses sintomas após a realização de variadas formas de terapias para o tratamento dos DTM, sugerindo novos estudos com grupos controle, a fim de que se possa avaliar a correta etiologia dessa relação.
O presente estudo verificou uma melhora significativa do zumbido após a realização de tratamentos conservadores para DTM. Dentre os fatores que possam justificar tal resultado, podemos destacar que a nossa casuística possui uma média de idade mais baixa, com mínima exposição a ruídos e presença de doenças crônicas e poucos indivíduos com alterações audiométricas, o que deve ter reduzido a presença de outras afecções como possíveis causa de zumbido, além dos DTM que devem ser valorizados como provável etiologia isolada ou concomitante.

\section{REFERÊNCIAS BIBLIOGRÁFICAS}

1. Silveira AM, Feltrin PP, Zanetti RV, Mautoni MC. Prevalência de portadores de DTM em pacientes avaliados no setor de otorrinolaringologia. Rev Bras Otorrinolaringol. 2007, 73(4):528-32.

2. BrutoLH, Kós AOA, Amado SM, Monteiro CR, Lima MATd. Alterações Otológicas nas Desordens TêmporoMandibulares. Rev Bras de Otorrinolaringol. 2000, 66(4):32732.

3. Urban VM, Neppelenbroek KH, Pavan S, Alencar Junior FGPd. Associação entre otalgia, zumbido, vertigem e hipoacusia com desordens temporomandibulares. RGO. 2009, 57(1):107-15.

4. Costen JB. A syndrome of ear and sinus symptoms dependent upon disturbed function of the temporomandibular joint. Ann of Otol Rhinol and Laryngol. 1934, 43:1-15.

5. Ramírez LM, Sandoval GP, Ballesteros LE. Los desordenes temporomandibulares: clinica craneo-cervicofacial referida. Med Oral Patol Oral Cir Bucal. 2005, 10:E18-E26.

6. RamírezLM, Sandoval GP, Ballesteros LE. Síntomas óticos referidos en desórdenes temporomandibulares.Relación con músculos masticatorios. Rev Méd Chile. 2007, 135:158290 .

7. Felício CMd, Faria TG, Silva MAMRd, Aquino AMCMd, Junqueira CA. Desordem temporomandibular: relações entre sintomas otológicos e orofaciais. Rev Bras Otorrinolaringol. 2004, 70(6):786-93.

8. Pascoal MIN, Rapoport A, Chagas JFS, Pascoal MB, Costa CC, Magna LA. Prevalência dos sintomas otológicos na desordem temperomandibular: estudo de 126 casos. Rev Bras Otorrinolaringol. 2001, 67(5):627-33.

9. Parker WS, Chole RA. Tinnitus, vertigo, and 
temporomandibular disorders. Am J Orthod Dentofac Orthop. 1995, 107(2):153-8.

10. De Felício C, Melchior M, Ferreira C, Rodrigues Da Silva M. Otologic symptoms of temporomandibular disorder and effect of orofacial myofunctional therapy. Cranio. 2008, 26(2):118-25.

11. Tuz H, Onder E, Kisnisci R. Prevalence of otologic complaints in patients with temporomandibular disorder. Am J Orthod Dentofacial Orthop. 2003, 123(6):620-3.

12. Garanhani RR. Sintomas otológicos na disfunção craniomandibular. [Monografia]. Florianópolis: Universidade Federal de Santa Catarina, Curso de Odontologia; 1998.

13. Mota LAA, Albuquerque KMGd, Santos MHP, Travassos RdO. Sinais e Sintomas Associados à Otalgia na Disfunção Temporomandibular. Arq Int Otorrinolaringol. 2007, 11(4):411-5.
14. Wright EF, Syms CA 3rd, Bifano SL. Tinnitus, dizziness, and nonotologic otalgia improvement through temporomandibular disorder therapy. Mil Med. 2000, 165(10):733-6.

15. Wright E, Bifano S. Tinnitus improvement through TMD therapy. J Am Dent Assoc 1997; 128: 1424-32.

16. Torii K, Chiwata I. Occlusal management for a patient with aural symptoms of unknown etiology: a case report. Journal of Medical Case Reports. 2007, 1:85.

17. Bösel C, Mazurek B, Haupt H, Peroz I. Chronischer Tinnitus und kraniomandibuläre Dysfunktionen: Einfluss funktionstherapeutischer Maßnahmen auf die Tinnitusbelastung. HNO. 2008, 56:707-13. 